\title{
La restauración ambiental de ecosistemas deforestados por cultivos ilícitos, el nuevo desafío del Ejército Nacional
}

\section{Environmental Restoration of Ecosystems Deforested by Illegal Crops, the New Challenge for the National Army}

Juliana Jiménez Velandia', Lonis Alberto Vega Delgado

\section{Resumen}

Durante los últimos años, el incremento de los cultivos ilícitos y la disputa por el control, uso y aprovechamiento de los recursos naturales han sido el escenario en el que se viene desarrollando el conflicto armado en el país; lo anterior, sumado a las acciones terroristas de grupos al margen de la ley, han desencadenado una problemática socioambiental profunda que ha contribuido con la deforestación, la degradación de ecosistemas naturales y el incremento de problemáticas asociadas a la violencia y el narcotráfico. En esta perspectiva, el presente artículo pretende evaluar y analizar los principales desafíos que en el marco del posconflicto debe asumir el Ejército Nacional, para contribuir en los procesos de recuperación y restauración ambiental de territorios deforestados por cultivos ilícitos. Por esta razón, se ha establecido como zona de observación el área del Catatumbo - Norte de Santander, en donde mediante la revisión bibliográfica de artículos de investigación, informes nacionales e internacionales de monitoreo, seguimiento y evaluación de cultivos de coca en Colombia por entidades gubernamentales y no gubernamentales, se identificaron las principales estrategias a adoptar para la rehabilitación ecológica de territorios disturbados. De acuerdo con lo anterior, se concluyó que dentro de las principales estrategias

1 Especialización Gestión Ambiental y Desarrollo Comunitario, Centro de Educación Militar - CEMIL, Bogotá.

Correo: julianajimenez112囚amail.com 
que se establecieron se incluyen: la protección del medio ambiente a través del desarrollo de actividades de reforestación y recuperación de servicios ecosistémicos, las actividades de defensa y soberanía del territorio mediante el acompañamiento y despliegue de tropas, así como el desarrollo de operaciones militares enfocadas exclusivamente a la rehabilitación y restauración ambiental.

Palabras clave: cultivos ilícitos, deforestación, gestión socioambiental, recuperación ambiental, posconflicto.

\section{Abstract}

Recently, the increase in illicit crops and the dispute over the control, use, and exploitation of natural resources have been the setting for the country's armed conflict. Added to terrorist actions by subversive groups, this situation has unleashed a severe socio-environmental problem that has aggravated deforestation, the degradation of natural ecosystems, violence, and drug trafficking. From this perspective, this article aims to assess and discuss the significant challenges that the National Army must take on in the post-conflict context to help in the environmental recovery and restoration of territories deforested by illicit crops. Catatumbo, Norte de Santander, was established as the area of observation. We identified the main strategies for the ecological rehabilitation of disturbed territories through a literature review of research articles and national and international reports on the monitoring, follow-up, and assessment of coca crops in Colombia by governmental and non-governmental organizations. In brief, these strategies include the protection of the environment through reforestation and recovery of eco-systemic services, defense and sovereignty of the territory by troop support and deployment, and military operations focused only on environmental rehabilitation and restoration.

Keywords: Illicit crops, deforestation, socio-environmental management, environmental recovery, post-conflict. 


\section{Introducción}

En Colombia, la disputa por el aprovechamiento de los recursos naturales ha sido uno de los principales motivos del conflicto que se ha desencadenado desde hace más de cincuenta años por grupos al margen de la ley, siendo este una causa importante del deterioro ambiental, el desplazamiento humano y la explotación descontrolada de los recursos naturales como consecuencia de actividades ilícitas como el narcotráfico, la minería, la deforestación y el tráfico ilegal de especies silvestres.

Actualmente, con el Acuerdo de Paz que tuvo lugar el 26 de septiembre de 2016, los procesos de recuperación y restauración ambiental de los territorios, donde se desarrolló el conflicto, y que hoy viven las consecuencias de la guerra, se consideran por parte del Estado, temas prioritarios para desarrollar programas estratégicos de conservación y mitigación en los ecosistemas impactados. En esta perspectiva, el ambiente, comprendido como una de las dimensiones del desarrollo sostenible, se asume como uno de los retos para garantizar la estabilidad de los ecosistemas, el desarrollo de las regiones y el mejoramiento de la calidad de vida en el país. Desde el marco de este escenario, el presente documento pretende evaluar y analizar los principales desafíos institucionales y misionales que el Ejército Nacional de Colombia asume como uno de los actores tanto del conflicto como del pos-Acuerdo, en las dinámicas de recuperación y restauración ambiental de zonas deforestadas por cultivos ilícitos.

Para efectos del desarrollo de la temática propuesta, se estableció como zona de estudio, las problemáticas de deforestación asociadas a la siembra de cultivos ilícitos de coca (Erythroxylum coca) que se presentan en la zona del Catatumbo (Norte de Santander). Para ello, se ha dispuesto de instrumentos de análisis comparativos, con el fin de obtener información veraz, clara y coherente sobre las particularidades culturales, ambientales, políticas, sociales y económicas de la zona, con el propósito de plantear estrategias sostenibles oportunas que promuevan e incentiven la participación del Ejército Nacional en la resolución de esta problemática socioambiental.

El análisis de esta información permitió desarrollar un ejercicio de reflexión sobre los resultados obtenidos a partir de las medidas de tipo social, de defensa y de gestión ambiental que debe desarrollar el Ejército Nacional de Colombia en aras al fortalecimiento de las actividades 
de control y vigilancia, y la consolidación de institucionalidad militar, la cual en un principio se enfocó en la defensa de la soberanía nacional, pero que ahora tiene como reto principal la restauración y recuperación de ecosistemas y los recursos naturales que lo conforman.

\section{Antecedentes}

Colombia es uno de los países con mayor diversidad biológica, cuenta con una gran variedad de ecosistemas estratégicos, dentro de los cuales se encuentran bosques aluviales de vegas, bosques húmedos tropicales, bosques secos o subhúmedos tropicales, así como especies de fauna y flora que lo catalogan como un país megadiverso (Andrade, 2011). Sin embargo, en los últimos años ha sido evidente el incremento en la degradación ambiental producto de la deforestación como consecuencia de los cultivos ilícitos; estas actividades lideradas en su mayoría por grupos al margen de la ley, se han extendido a todas las regiones del país, afectando principalmente territorios de gran importancia ambiental y ecosistémica, tal es el caso de las zonas selváticas de la región del Catatumbo (Norte de Santander).

La apropiación de tierras, los cultivos ilícitos, la minería ilegal y el incremen- to de incendios forestales, son para el Estado colombiano las principales causas de la problemática masiva a combatir. El flagelo que se estima que en el 2018 erradicó más de 270000 hectáreas de bosque (Ministerio de Ambiente y Desarrollo Sostenible, 2019). Esta explotación ilícita y descontrolada de recursos naturales ha ocasionado un deterioro ambiental que ha generado efectos adversos en la biodiversidad del país.

De la misma manera, el Instituto de Hidrología, Meteorología y Estudios Ambientales (Ideam), por medio del Sistema de Monitoreo de Bosques y Carbono (SMBC), expuso el aumento del $44 \%$ en la deforestación nacional en el 2017, en comparación con el 2015, al pasar de 124035 a 178597 hectáreas de bosques destruidos (Ideam, 2017). Este aumento en la deforestación del país enciende las alarmas nacionales, e impide el cumplimiento de los Objetivos de Desarrollo Sostenible que se ha propuesto el país en la mitigación de los efectos del cambio climático y en la protección de los ecosistemas terrestres para el 2030.

Otro estudio realizado por la Oficina de las Naciones Unidas contra la Droga y el Delito (UNODC, por sus siglas en inglés) en el 2018 indicó que: 
Tabla 1. Hectáreas de coca (Erythroxylum coca) cultivadas en Colombia, 2012-2019

\begin{tabular}{|c|c|c|c|c|c|c|c|c|c|}
\hline Departamento & $\mathbf{2 0 1 2}$ & $\mathbf{2 0 1 3}$ & $\mathbf{2 0 1 4}$ & $\mathbf{2 0 1 5}$ & $\mathbf{2 0 1 6}$ & $\mathbf{2 0 1 7}$ & $\mathbf{2 0 1 8}$ & $\mathbf{2 0 1 9}$ & $\begin{array}{c}\text { \% } \\
\text { cambio } \\
\mathbf{2 0 1 8} \\
\mathbf{2 0 1 9}\end{array}$ \\
\hline Nariño & 10733 & 13177 & 17285 & 29755 & 42627 & 45734 & 41903 & 36964 & \\
\hline Putumayo & 6148 & 7667 & 13609 & 20068 & 25162 & 29588 & 26407 & 24972 & \\
\hline $\begin{array}{c}\text { Norte de } \\
\text { Santander }\end{array}$ & $\mathbf{4 5 1 6}$ & $\mathbf{6 3 4 5}$ & $\mathbf{6 9 4 4}$ & $\mathbf{1 1 5 2 7}$ & $\mathbf{2 4 8 3 1}$ & $\mathbf{2 8 2 4 4}$ & $\mathbf{3 3 5 9 7}$ & $\mathbf{4 1 7 1 0}$ & $\mathbf{2 4 , 1 4 \%}$ \\
\hline Cauca & 4325 & 3326 & 6389 & 8660 & 12595 & 15960 & 17117 & 17355 & \\
\hline Caquetá & 3695 & 4322 & 6542 & 7712 & 9343 & 11793 & 11761 & 4511 & \\
\hline
\end{tabular}

Fuente: Oficina de las Naciones Unidas Contra la Droga y el Delito (UNODC), 2020.

Monitoreo de territorios afectados por cultivos ilicitos. http://www.odc.gov.co/sidco/oferta/cultivos-ilicitos/departamento-municipio.

Fuente: elaboración propia.

Los cultivos de coca en Colombia, desde 2013, han incrementado a un ritmo del $45 \%$ anual, pasando de 48.000 ha en 2013 a 146.000 ha en 2016. Este incremento constante sucedió en el marco de las negociaciones de la paz con las Fuerzas Armadas Revolucionarias de Colombia (FARC) y en una transición en la estrategia de lucha contra los cultivos de coca. A 2017, el área sembrada con coca tuvo un incremento del $17 \%$ con respecto al año anterior, el $64 \%$ del incremento se encuentra en los departamentos de Putumayo, Norte de Santander y Cauca. (UNODC, 2018)

El crecimiento de cultivos ilícitos se observa en la tabla 1. Hectáreas de coca
(Erythroxylum coca) cultivadas en6 Colombia durante el periodo comprendido entre el 2008 y 2016 en varios departamentos del país, desarrollado por la Oficina de las Naciones Unidas Contra la Droga y el Delito (2017).

De acuerdo con la tabla 1, la siembra de cultivos ilícitos en el departamento de Norte de Santander presenta un crecimiento exponencial del $115 \%$ entre el 2015 y 2016. En otro estudio, el Observatorio de Drogas de Colombia (2018) indicó que para el 2018 el área sembrada por cultivos ilícitos de coca en el Norte de Santander fue de 33597 hectáreas en comparación con 24831 hectáreas sembradas en el 2016, reportadas por 
la UNODC. Lo que indica que, a pesar de los esfuerzos del Estado por implementar el Acuerdo de Paz y desarrollar programas de sustitución de cultivos ilícitos, los resultados no han sido completamente exitosos.

Según la información proporcionada a partir de los reportes técnicos, es posible inferir que el incremento de los cultivos de coca en el país está relacionado con el aumento de los precios de la base de coca en el mercado ilegal, los cambios en la política de fumigación y la injerencia que vienen teniendo algunos grupos armados en el país (El Universal, 2018).

En la figura 1 se presenta la concurrencia de los cultivos ilícitos, conflicto armado y deforestación, así como los tipos de cobertura del departamento de Norte de Santander.

Figura 1. Concurrencia de cultivos ilícitos, conflicto armado y deforestación, 2016

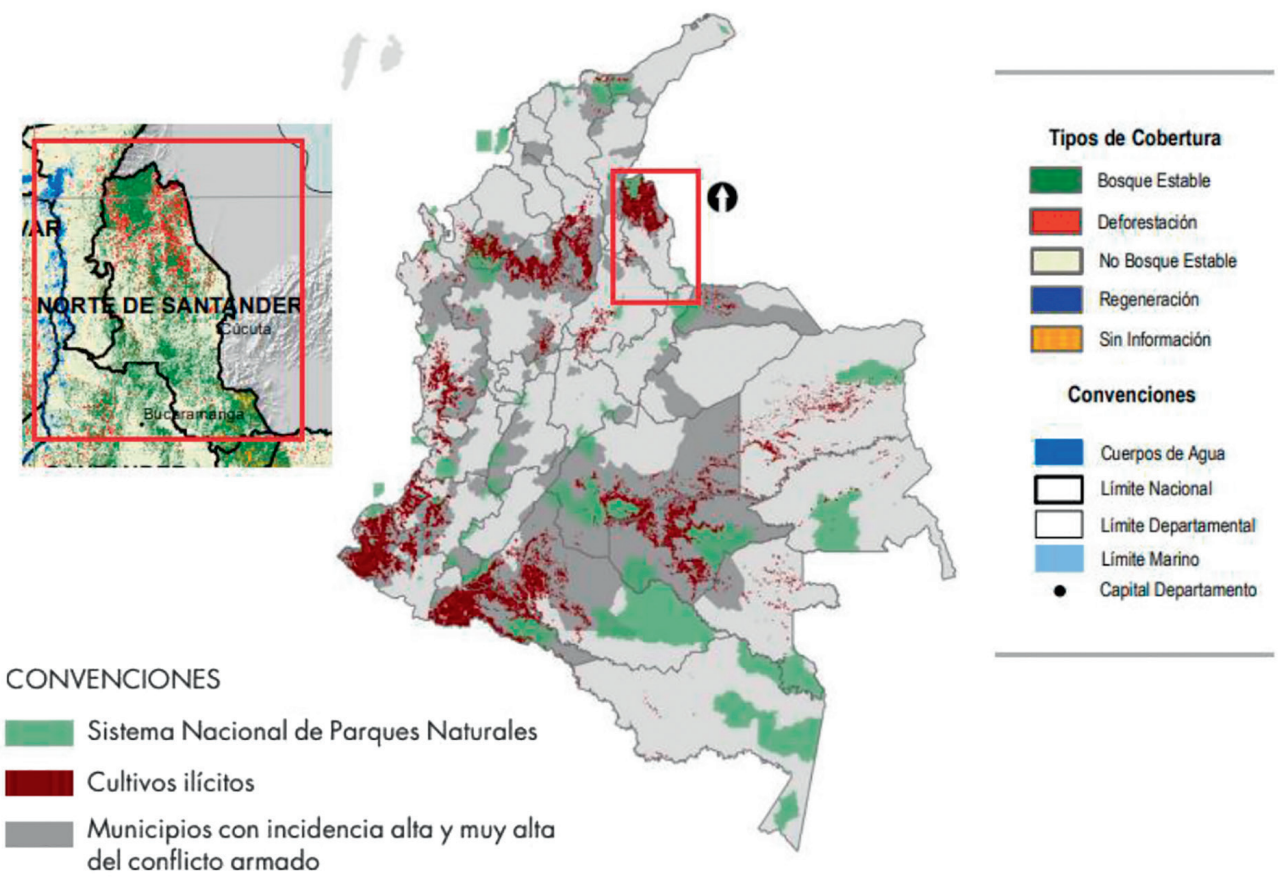

Fuente: Departamento Nacional de Planeación (DNP), 2016. Panorámica regional dividendos ambientales de la paz. https://colaboracion.dnp.gov.co/CDT/Prensa/Publicaciones/PANORAMICA\%20VERSI\%C3\%93N\%20FINAL\%2019\%20enero2017.pdf Autor: DNP (2016). 
La relación entre el crecimiento de cultivos ilícitos y el conflicto armado se observa en la figura 1. Mapa de la concurrencia de cultivos ilícitos, conflicto armado y deforestación desarrollado por el Departamento Nacional de Planeación (2016), en donde se observa cómo dentro de los siete motores de deforestación identificados, cuatro están estrechamente ligados al conflicto: cultivos ilícitos, extracción ilícita de minerales, extracción ilegal de madera y desplazamiento.

Este aumento en la siembra de cultivos ilícitos ha ocasionado alteraciones negativas sobre el ambiente. El Ministerio de Ambiente y Desarrollo Sostenible (2019) indicó que

La deforestación asociada a cultivos de coca tiene impactos relacionados con la pérdida y migración de la fauna y flora endémica de estos bosques, pérdida de estos suelos, aumento de la erosión, desertificación, y el consecuente incremento de deslizamientos, avalanchas y demás desastres asociados en el país.

De igual manera, según información proporcionada por la Dirección de Antinarcóticos de la Policía Nacional (2014), los cultivos de coca, así como el procesamiento de transformación de la hoja de coca a clorhidrato de cocaína, gene- ran vertimientos derivados de la utilización intensiva de fertilizantes, que, por la naturaleza ilícita de la actividad, no son compensados ni mitigados.

En consecuencia con lo anterior, estas afectaciones al ambiente comprometen de manera significativa las dinámicas socioculturales y económicas de un territorio. Este es el caso de la zona del Catatumbo, donde activistas y organizaciones defensoras del ambiente insisten en que la expansión de los cultivos ilícitos sigue agotando la riqueza natural de la subregión del Catatumbo y atentando contra la integridad, calidad de vida y seguridad de la población (El Tiempo, 2018).

Teniendo en cuenta lo anterior, la Organización de las Naciones Unidas (ONU, 2018) aseguró que las disparidades en el acceso a los derechos económicos, sociales, culturales y ambientales son mayores en los municipios más afectados por el conflicto armado. Por ejemplo, los municipios considerados por el Gobierno como zonas más afectadas por el conflicto armado tienen, en promedio, un índice de pobreza multidimensional del $76 \%$, cuando la media nacional es del $49 \%$.

Asimismo, este informe reveló que a esta problemática socioambiental la acompaña también hechos de criminalidad 
y violencia, como puede evidenciarse en los catorce casos de defensores y líderes desplazados fuera del Catatumbo ante amenazas por su labor de impulso al Programa Nacional de Sustitución de Cultivos de Uso Ilícito (PNIS) en el 2018, en donde se encontró que:

Los presuntos autores de estos hechos fueron principalmente miembros de organizaciones criminales, entre cuyos integrantes se encuentran posiblemente antiguos miembros de estructuras paramilitares desmovilizadas (40\%), individuos no afiliados a ningún grupo criminal o grupo armado ilegal (18\%), integrantes del ELN (8\%), integrantes del Ejército Popular de Liberación (EPL) (4\%), antiguos miembros de las FARC-EP que no se acogieron al proceso de paz $(8 \%)$ y personas por determinar (22\%). (ONU, 2018)

\section{Marco teórico}

La deforestación en el trópico se reconoce como un problema socioecológico complejo que ha sido explorado desde diferentes perspectivas disciplinares, especialmente desde el punto de vista económico, social, institucional y ambiental (Angelsen, 1999). En el ámbito nacional, la pérdida de bosques es una de las principales amenazas de la diversidad biológica y la pérdida de servicios ecosistémicos.

En Colombia, la problemática de la deforestación ha estado íntimamente relacionada con el crecimiento y expansión de cultivos ilícitos de Erythroxylum coca. Según la Dirección Nacional de Estupefacientes de Colombia (2008), los cultivos ilícitos tienen efectos sobre el medio natural, los primeros efectos ambientales inician con la destrucción de la flora nativa, el agotamiento de la materia orgánica de los suelos de vocación forestal, seguido por la generación de procesos erosivos y la destrucción de las cadenas tróficas y nichos ecológicos.

Desde el punto de vista ambiental, la tala y quemas de los bosques, como consecuencia de la siembra de cultivos ilícitos, hacen que los suelos que han sido desprotegidos de la cobertura vegetal inicien procesos de erosión que generan su desestabilización y favorecen el proceso de remoción en masa (Dirección Nacional de Estupefacientes, 2008). Este tipo de prácticas se ha convertido en una amenaza ambiental, afectando las fuentes de reserva hídrica debido a la deforestación que se presenta en estos espacios que son convertidos en llanuras, cuyo fin no corresponde a la función 
de acumulación y protección del agua, propias de estos espacios ecológicos.

De la misma forma, según este estudio realizado por la Dirección Nacional de Estupefacientes (DNE, 2008), para implementar una hectárea de coca los cultivadores tienen que destruir cuatro hectáreas de selva. En un primer acercamiento al estudio de impactos ambientales ocasionados por la producción y procesamiento del cultivo de coca, Ramos y Ramos (2002, pág. 10) concluyen que el uso de agroquímicos durante los primeros cinco años de cultivo es de 1,7 veces más en el cultivo de coca que en el de cacao, y que el uso de pesticidas en el establecimiento del cultivo es diez veces mayor al usado en el cultivo de cacao. De igual manera, por el procesamiento para obtener un kilo de pasta básica de cocaína, se vierten en el ambiente 1,9 L de ácido sulfúrico, 1,25 L de amoníaco, 193,75 L de agua contaminada y $625 \mathrm{~kg}$ de residuos vegetales.

El informe sobre el censo de cultivos de coca desarrollado por la UNODC (2006, pág. 63), calculó que para las 85770 ha cultivadas de coca en el país en el 2005, se demandaron 81770 toneladas métricas de insumos para el procesamiento como gasolina y cemento, y 13 millones de litros entre fertilizantes, herbicidas y pesticidas, algunos de los cuales se encuentran en la categoría de altamente tóxicos.

La Organización de las Naciones Unidas para la Alimentación y la Agricultura (FAO), asegura que el exceso de agroquímicos puede causar a largo plazo la infertilidad del suelo con el aumento de la acidez de los mismos, así como la contaminación de las aguas subterráneas, que pueden ser arrastradas a cursos de agua, provocando con esta sobrecarga de nutrientes la eutrofización de lagos, embalses y estanques, así como la contaminación de los suministros de agua potable (FAO, 2018).

Del mismo modo, el problema ambiental ocasionado por la deforestación y los cultivos ilícitos se convierte en un desafío social que afecta de manera negativa la seguridad de la población y que propicia el incremento de la criminalidad, el narcotráfico y la violencia. Por lo cual, debe analizarse desde múltiples perspectivas y debe involucrarse a los distintos actores gubernamentales, no gubernamentales, actores sociales, autoridades ambientales locales, institutos de investigación y fuerzas militares de Colombia.

\section{Metodología}

La presente metodología se basa en un ejercicio de revisión bibliográfica de 
informes técnicos de orden nacional e internacional, especializados en temas de monitoreo, seguimiento y evaluación de los impactos socioambientales generados por los cultivos de coca en Colombia. Para ello, se han considerado los reportes elaborados por entidades gubernamentales, como el Ministerio de Ambiente y Desarrollo Sostenible, la Dirección Nacional de Estupefacientes de Colombia y la Oficina de Naciones Unidas contra la Droga y el Delito, con una ventana de observación de tiempo de los últimos diez años (2009-2019).

Igualmente, se analizaron diversos informes desarrollados por organizaciones no gubernamentales (ONG) como la Fundación Ideas para la Paz y la ONG Human Rights Watch, las cuales en el 2019 desarrollaron encuestas de tipo social a más de ochenta actores, incluidos residentes de zonas rurales y urbanas del Catatumbo, funcionarios judiciales, funcionarios de derechos humanos, autoridades locales, líderes sociales y miembros de organizaciones humanitarias y de derechos humanos sobre la situación socioambiental de la región del Catatumbo, Norte de Santander.

Asimismo, se analizaron artículos e informes científicos, suministrados por institutos de investigación científica y organismos especializados de la ONU, como el Ideam, el mecanismo para la Reducción de las Emisiones de la Deforestación y la Degradación de Bosques (REDD+), el Sistema Integrado de Monitoreo de Cultivos Ilícitos (SIMCI) y la FAO, respectivamente; con el propósito de obtener y comparar las cifras relacionadas con la dinámica de los cultivos de coca en el país, durante los últimos años, y analizar sus impactos ambientales ocasionados sobre los ecosistemas de selva húmeda tropical.

La información que se obtuvo a partir de esta revisión bibliográfica se analizó de forma comparativa, con el propósito de identificar estrategias sostenibles que permitan incrementar la efectividad de las políticas nacionales de erradicación de cultivos ilícitos e involucrar al Ejército Nacional en la recuperación, conservación y reforestación de los territorios ambientalmente degradados por esta problemática.

De acuerdo con lo anterior, se escogieron como criterios de evaluación los impactos socioambientales ocasionados por los cultivos ilícitos en el Catatumbo, que incluyen: deforestación, pérdida de servicios ecosistémicos, contaminación del recurso hídrico, pérdida de flora y fauna, afectaciones sociales generadas en 
la comunidad a raíz de la criminalidad; así como la evaluación de la efectividad y viabilidad de las iniciativas gubernamentales, como el Programa de Sustitución de Cultivos Ilícitos y el Plan Artemisa, cuyo objetivo es detener la deforestación y recuperar los ecosistemas boscosos del país.

\section{Resultados}

A partir de la revisión bibliográfica realizada, se evidenció que el índice de degradación ambiental ocasionado por la deforestación es mayor en los departamentos que tienen presencia de cultivos ilícitos, según la Dirección de Antinarcóticos (2014). Lo anterior se debe, principalmente, al acaparamiento de tierras (45\%), el desarrollo de cultivos ilícitos $(22 \%)$, expansión de infraestructura $(10 \%)$, incendios forestales ( $8 \%$ ), ganadería extensiva $(8 \%)$ y minería mecanizada e ilegal (7\%).

En comparativa, los informes científicos sobre los impactos ambientales ocasionados por la deforestación emitidos por el Ideam (2017) y la FAO (2018), concuerdan en que la degradación ambiental ocasionada por la siembra de cultivos ilícitos ha traído como consecuencia inmediata la erosión y pérdida de diversidad biológica. Para los suelos donde el uso intensivo ha producido agotamiento, los cultivadores de coca han debido utilizar agroquímicos a fin de compensar la pérdida de fertilidad natural y las malas prácticas ambientales que se desarrollan en este tipo de actividades; lo que se ha traducido en la búsqueda de nuevas áreas para la siembra de coca y en un aumento de la gravedad e intensidad de esta problemática socioambiental (DIRAN, 2014).

Esta creciente degradación ambiental está íntimamente relacionada con la efectividad que ha tenido la implementación del programa de sustitución de cultivos ilícitos en el país, el cual esboza ciertas limitaciones en el cumplimiento de sus objetivos. Según el análisis desarrollado, a partir de los informes técnicos emitidos por el Ministerio de Ambiente y Desarrollo Sostenible (2016) y la Oficina de las Naciones Unidas contra la Droga y el Delito (2018), este incremento en la siembra de cultivos ilícitos indica que se requiere de la implementación de estrategias sostenibles, que integren las dimensiones social, ambiental, económica, política y cultural, a fin de asumir alternativas efectivas y transversales en el corto, mediano y largo plazo, comprometiendo todas las voluntades tanto comunitarias como institucionales, e involucrando a las Fuerzas Militares, donde el Ejército Nacional desempeña una función esencial. 


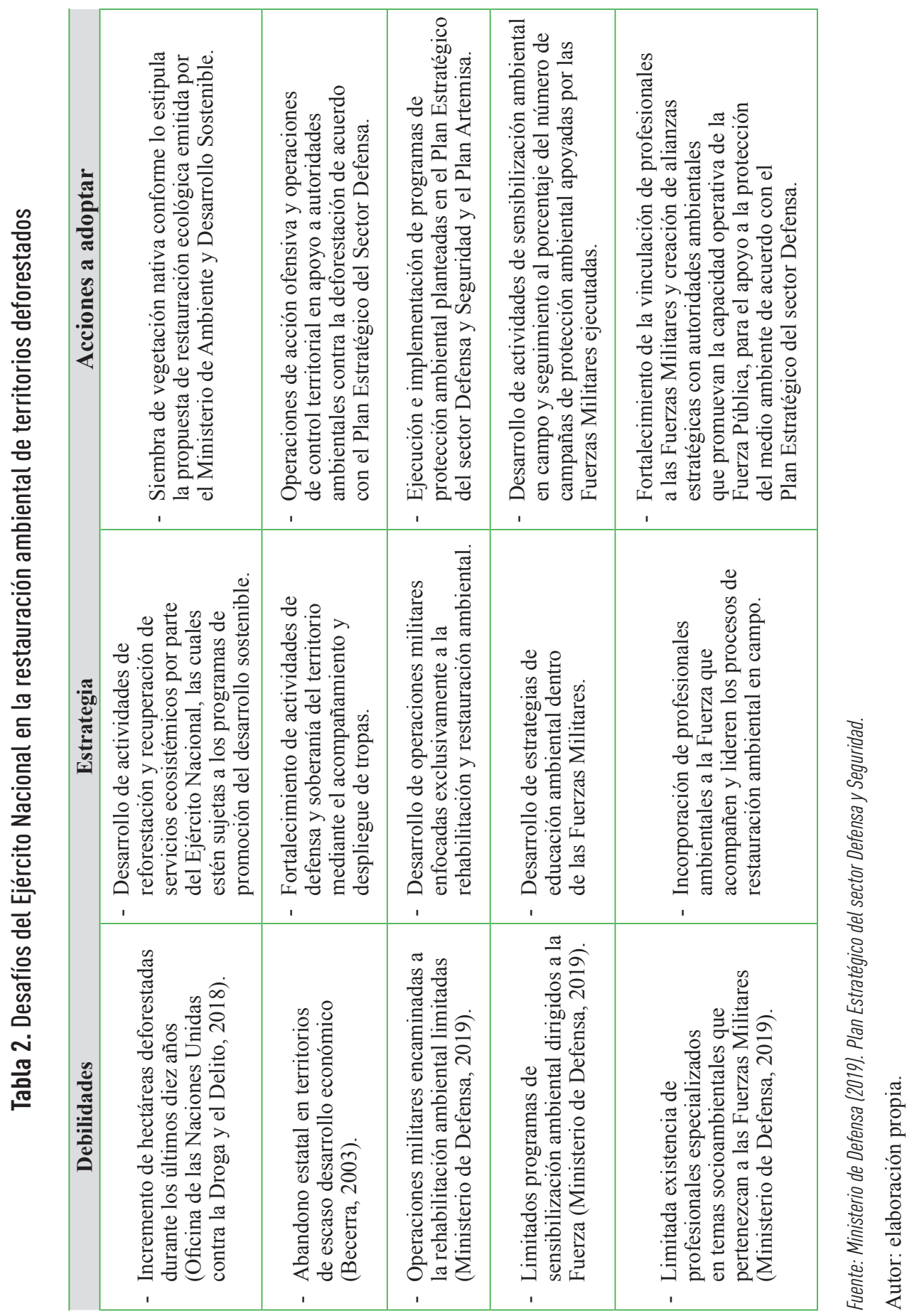


De acuerdo con lo anterior, con el actual "Plan Artemisa", emitido a principios del 2019, se abren nuevas oportunidades para que el Ejército Nacional participe activamente en la recuperación de territorios deforestados por actividades ilícitas y contribuya en la ejecución de estrategias que contemplen la protección y restauración ambiental de territorios que han sufrido las secuelas del conflicto armado. Desde esta premisa, la fuerza debe asumir este desafío integrando esfuerzos operacionales, misionales y de planeación.

Por lo cual, a partir de la revisión bibliográfica se identificaron las principales debilidades gubernamentales e institucionales que han conllevado un crecimiento progresivo de los cultivos ilícitos, y se proponen una serie de estrategias y acciones a desarrollar por parte del Ejército Nacional en aras de salvaguardar, proteger y restaurar los ecosistemas selváticos, como se expresa en la tabla 2.

Las estrategias anteriormente planteadas apuntan hacia el fortalecimiento de la creación de unidades militares que se dediquen específicamente a la labor de gestión ambiental, donde exista una visión renovada, y se atienda los retos actuales que en materia ambiental y seguridad ecológica se presentan en el país.
De esta forma, el Ejército Nacional como fuerza multimisión, no solo debe asegurar los intereses del Estado desde una perspectiva defensiva, sino también desde una perspectiva socioambiental, mediante el despliegue de esfuerzos en pro del fortalecimiento de estrategias de sostenibilidad en zonas como el Catatumbo, que requieren con urgencia del despliegue militar y del desarrollo de actividades de reforestación, protección de flora y fauna silvestre, control de actividades ilícitas, apoyo a entidades ambientales e incremento de programas de sensibilización.

\section{Conclusiones}

Colombia se encuentra actualmente en un escenario de pos-Acuerdo, lo que conlleva la necesidad de reflexionar, estructurar, planear y consolidar espacios de paz, que giren en torno a la importancia del cuidado y recuperación del territorio; pues es alrededor de este en donde la sociedad reformula su sentido de pertenencia, replantea su arraigo cultural y puede reconocer su entorno natural, ya no mediante la perspectiva de dominio, sino mediante la armonía y el respeto. Por tanto, debe considerarse que la defensa y seguridad no solo son de orden social y económico, sino también ambiental. 
El Ejército Nacional, como fuerza legítima de Colombia, cuya misión principal es la de defensa de la soberanía, el orden constitucional y la integridad del territorio nacional, está llamado a desempeñar un papel esencial en la transformación de los conflictos socioambientales relacionados con actividades ilícitas. Junto al papel protagonista del Estado, las Fuerzas Militares de Colombia deben ejercer la gobernanza de los recursos naturales, desde un diálogo multiactoral que involucre las distintas voces y que sea encaminado bajo el principio de la responsabilidad compartida, unificando esfuerzos en escenarios bilaterales en aras de conciliar las relaciones hoy transgredidas entre la sociedad y la naturaleza.

\section{Referencias}

Andrade-C., M. G. (2011). Estado del conocimiento de la biodiversidad en Colombia y sus amenazas. Consideraciones para fortalecer la interacción ambiente-política. Rev. Acad. Colomb. Cienc., 35(137), 491507, http://www.scielo.org.co/pdf/racefn/ v35n137/v35n137a08.pdf

Angelsen, A. (1999). Expansión agrícola y deforestación: modelando el impacto de la población, fuerzas del mercado y derechos de propiedad. Revista de Economía del Desarrollo, (58), 185-218.
Bannon, I. (2003). Recursos naturales y conflicto violento: opciones y acciones. Washington: The World Bank.

Becerra, M. (2003). Los cultivos ilícitos y el medio ambiente. Presentado en la VIII Cátedra Anual de Historia "Ernesto Restrepo Tirado", Análisis histórico del narcotráfico en Colombia. http://www.manuelrodriguezbecerra.org/bajar/ilicitos.pdf

Comando General de las Fuerzas Militares. (2016). El medio ambiente, una prioridad para las Fuerzas Militares. En: La Fuerza, No. 40. http://www.cgfm.mil.co/ wp-content/uploads/2017/05/40-Las-Fuerzas-2016.pdf

Defensoría del Pueblo. (2006). Situación social $y$ ambiental de la región del Catatumbo, Norte de Santander. file://C:/Users/USER/ Downloads/defensorial46\%20(1).pdf

Defensoría del Pueblo. (2014). El riesgo de reclamar la tierra. Vulneración y amenazas a los derechos humanos de personas y comunidades. Bogotá: Defensoría del Pueblo.

Defensoría del Pueblo. (2016). Análisis de contexto y definición de escenarios de riesgo. Bogotá: Sistema de Alertas Tempranas-Equipo Humanitario Local Putumayo.

Delgado, C. (2008). ¿Qué retos plantea a la filosofia la problemática ambiental? Tesis presentada por el acto de celebración del día internacional de la filosofía. La Habana.

Departamento Nacional de Planeación (DNP). (2016). Panorámica regional. Dividendos ambientales de la paz. https:// colaboracion.dnp.gov.co/CDT/Prensa/ Publicaciones/PANORAMICA\%20VERSI\%C3\%93N\%20FINAL\%2019\%20enero2017.pdf 
Dirección Nacional de Estupefacientes (DNE). (2008). Impacto ambiental ocasionado por las sustancias químicas, los cultivos ilicitos y las actividades conexas. http://www. dne.gov.co/?idcategoria $=790$

Dirección de Antinarcóticos (DIRAN). (2014). COCA: deforestación, contaminación y pobreza. http://www.odc.gov.co/Portals/1/publicaciones/pdf/oferta/estudios/ OF5022014-coca-deforestacion-contaminacion-pobreza.pdf

Dirección Estratégica de Medio Ambiente. (2017). Misión del proceso de gestión ambiental. https://www.cgfm.mil.co/es

Echandía, C. y Cabrera, I. (2017). Madurez para la paz: evolución de la territorialidad y las estrategias en el conflicto armado colombiano. Bogotá: Universidad Externado de Colombia.

El Tiempo. (9 de julio de 2018). Denuncian que cultivos ilícitos siguen devorando bosques del Catatumbo. https://www. eltiempo.com/colombia/otras-ciudades/ deforestacion-por-cultivos-ilicitos-en-catatumbo-en-norte-de-santander-241244

El Universal. (20 de septiembre de 2018). Los factores que habrían incidido en el incremento de los cultivos ilícitos. https://www.eluniversal.com.co/colombia/los-factores-que-habrian-incidido-en-el-incremento-de-los-cultivos-ilicitos-288114-MUEU405382

FAO. (2018). Perspectivas para el medio ambiente. http://www.fao.org/3/y3557s/ y3557s11.htm
Fundación Ideas para la Paz. (2013). Dinámicas del conflicto armado en el Catatumbo y su impacto humanitario. Área de Dinámicas del Conflicto y Negociaciones de Paz - Boletín No. 64. http://cdn.ideaspaz.org/media/ website/document/5b72fe7f2b9d1.pdf

Fundación Ideas para la Paz. (2018). Desafios de la sustitución de cultivos y recomendaciones para el nuevo gobierno. http://www. ideaspaz.org/publications/posts/1696

Galano, C. (2005). Taller Preparatorio del 1er. Seminario Nacional de Educación Ambiental para el Desarrollo Humano Sustentable. Red Nacional de Educación Ambiental para el Desarrollo Humano Sustentable. Uruguay.

Human Rights Watch. (2019). Entrevista: La guerra en el Catatumbo colombiano. Colombianos y venezolanos quedan a merced de grupos armados. https://www.hrw.org/ es/news/2019/08/08/entrevista-la-guerra-en-el-catatumbo-colombiano

Human Rights Watch. (2019). La guerra en el Catatumbo. Abusos de grupos armados ilegales contra civiles colombianos y venezolanos en el noreste de Colombia. https:// www.hrw.org/es/report/2019/08/08/la-guerra-en-el-catatumbo/abusos-de-grupos-armados-contra-civiles-colombianos-y

Ideam. (2017). Cambio climático y deforestación. http://www.ideam.gov.co/web/ atencion-y-participacion-ciudadana/cambio-climatico 
Lavaux, S. (2004). Degradación ambiental y conflictos armados: las conexiones. Documento de investigación $\mathrm{N}^{\circ} 7$. https://pure.urosario.edu.co/ws/portalfiles/ portal/20316504/Degradaci_n_ambiental_y_conflictos_armados_las_conexiones_Stephanie.pdf

Massé, F. y Camargo, J. (2012). Actores armados ilegales y sector extractivo en Colombia. Bogotá: CIT Pax Colombia.

Mecanismo para la reducción de las emisiones de la deforestación. (2019). Bosques, territorios de vida. https://redd.unfccc.int/files/eicdgb bosques_territorios_de_vida_web.pdf

Ministerio de Medio Ambiente. (1993). "Por la cual se crea el ministerio del medio ambiente, se reordena el Sector Público encargado de la gestión y conservación del medio ambiente y los recursos naturales renovables, se organiza el Sistema Nacional Ambiental -SINA y se dictan otras disposiciones". http://www.humboldt.org.co/ images/documentos/pdf/Normativo/199312-22-ley-99-crea-el-sina-y-mma.pdf

Ministerio de Ambiente y Desarrollo Sostenible. (2019). Impactos de cultivos ilicitos en bosques de Colombia. http:// www.minambiente.gov.co/index.php/ noticias/4264-minambiente-llamo-la-atencion-sobre-impactos-de-cultivos-ilicitos-en-bosques-de-colombia

Ministerio de Defensa. (2019). Transformación y futuro de la fuerza pública 2010-2030. https://www.mindefensa.gov.co/irj/go/km/ docs/Mindefensa/Documentos/descargas/ estrategia_planeacion/proyeccion/documentos/trasnformacion_futuro_FP.pdf
Ministerio de Defensa. (2019). Plan Estratégico del Sector Defensa y Seguridad. https://www.mindefensa.gov.co/irj/go/km/ docs/Mindefensa/Documentos/descargas/ Sobre_el_Ministerio/Planeacion/Politicas/ PES_mar_2021.pdf

Molina, A. (2019). El papel de las Fuerzas Armadas en la protección y defensa del medio ambiente en Colombia. https://ceeep.mil. pe/2019/01/29/el-papel-de-las-fuerzas-armadas-en-la-proteccion-y-defensa-del-medio-ambiente-en-colombia/

Observatorio de Drogas de Colombia. (2018). Política de erradicación de cultivos de coca. http://www.odc.gov.co/POL\%C3\%8DTICA/Reducci\%C3\%B3n-de-la-oferta/ Erradicaci\%C3\%B3n

Oficina de las Naciones Unidas contra la Droga y el Delito. (2016). Monitoreo de territorios afectados por cultivos ilícitos. https:// www.unodc.org/documents/cropmonitoring/Colombia/Monitoreo_Cultivos_ilicitos_2015.pdf

ONU. (2018). Presentación en Cúcuta del Informe Anual de la Alta Comisionada de la ONU para los DD. HH. sobre Colombia. https://www.hchr.org.co/index.php/ informacion-ublica/pronunciamientos/ intervenciones-de-la-direccion/442-ano2019/9044-presentacion-en-cucuta-del-informe-anual-de-la-alta-comisionada-de-laonu-para-los-ddhh-sobre-colombia

Ramos, R. E. y Ramos, J. P. (2001). Evaluación ambiental del impacto de cultivos de coca y el proceso de la hoja. Universidad de los Andes, Bogotá. Colombia. 
Rangel, J. (2016). La biodiversidad en Colombia. Facultad de Ciencias Humanas, Universidad Nacional de Colombia. http:// www.bdigital.unal.edu.co/14263/1/38083-PB.pdf

Robayo, L. (2018). La guerra en el Catatumbo: abusos de grupos armados contra civiles colombianos y venezolanos en el noreste de Colombia. https://www.hrw.org/ es/report/2019/08/08/la-guerra-en-el-catatumbo/abusos-de-grupos-armados-contra-civiles-colombianos-y

Robayo, L. (2018). Entrevista: La guerra en el Catatumbo. Colombianos y venezolanos quedan a merced de grupos armados https://www.hrw.org/es/news/2019/08/08/ entrevista-la-guerra-en-el-catatumbo-colombiano

Rodríguez, C. y Durán, H. (2017). La paz ambiental, restos y propuestas para el posAcuerdo. Bogotá, Colombia. https://cdn. dejusticia.org/wp-content/uploads/ 2017/04/fi_name_recurso_924.pdf

Sistema Integrado de Monitoreo de Cultivos Ilícitos. (2010). Monitoreo de cultivos ilicitos. https://www.unodc.org/colombia/es/ censocultivos.html
UNODC. (2006). Caracteristicas agroculturales de los cultivos de coca en Colombia. Proyecto SIMCI. http://www.biesimci.org/ Documentos/archivos/Caracteristicas Agroculturales_Colombia_2006_Med.pdf

UNODC. (2011). Programa Familias Guardabosques. Datos análisis espacio temporal de coberturas 2003-2011. Bogotá.

UNODC. (2018). Colombia: incremento de $52 \%$ en los territorios afectados por cultivos de coca. https://www.unodc.org/ documents/colombia/2019/Agosto/Informe_de_Monitoreo_de_Territorios_Afectador_por_Cultivos_Ilicitos_en_Colombia_2018_.pdf

UNODC. (2018). Monitoreo de territorios afectados por cultivos ilícitos. https:// www.unodc.org/unodc/es/frontpage/2017/ July/new-unodc-report_coca-crops-in-colombia-increase-over-50-per-cent-in-oneyear.html

Vargas, J. (2012). Costos del conflicto y consideraciones económicas para la construcción de paz. En: Rettberg, A., Construcción de paz en Colombia (pp. 239-272). Bogotá: Universidad de los Andes. 